constrained fields in the elements of constructions. Kyiv: Scientific thinking, 280.

5. Ditkin, V. A., Prudnikov, A. O. (1965). Reference Book on an operating calculation. Moscow: Higher school, 466.
6. Pugachev, S. I. (Ed.) (1984). Pezokeramic transformers. Lviv: Shipbuilding, 256.

7. Slepan, L. I. (1972). Non-stationary resilient waves. Lviv: Shipbuilding, 374.

Рекомендовано до публікації доктортехнічних наук, професор Савін В. Г. Дата надходження рукопису 27.01.2015

Бабаев Александр Арташесович, кандидат физико-математических наук, доцент кафедры теоретической механики, Национальный технический университет Украины "Киевский политехнический институт", пр. Победы, 37, г. Киев, Украина, 03056

E-mail: babaevaa@ukr.net

Кришталь Владимир Федорович, кандидат технических наук, доцент кафедры теоретической механики, Национальный технический университет Украины "Киевский политехнический институт", пр. Победы, 37, г. Киев, Украина, 03056

E-mail:krishtalvf@gmail.com

Можаровская Тамара Николаевна, кандидат технических наук, доцент кафедры теоретической механики, Национальный технический университет Украины "Киевский политехнический институт", пр. Победы, 37, г. Киев, Украина, 03056

E-mail: mtn131954@ukr.net

УДК 537.32

DOI: 10.15587/2313-8416.2015.36332

\title{
ПЕРЕНОС ТЕПЛА ФОНОНАМИ В ТРАНСПОРТНОЙ МОДЕЛИ ЛАНДАУЭРА-ДАТТЫ-ЛУНДСТРОМА
}

\section{(C) Ю. А. Кругляк}

С позиичй транспортной модели ЛДЛ строится обобщенная модель переноса тепла фононами. Аналогично фермиевскому окну электронной проводимости вводится понятие фермиевского окна фононной проводимости и через него выводится общее выражение для решеточной теплопроводности, в котором с самого начала фигурирует квант теплопроводности. Подчеркивается подобие и различия 8 построении теории электронной проводимости и теории теплопроводности

Ключевые слова: нанофизика, наноэлектроника, фононный транспорт, квант теплопроводности, коэффициент прохождения, фононные моды, дебаевская модель, рассеяние фононов

On the basis of Landauer-Datta-Lundstrom transport model the generalized model of heat transfer by phonons is formulated. Similarly to the Fermi window for electron conductivity the concept of the Fermi window for phonon conductivity is introduced and used to obtain the general expression for the lattice thermal conductivity with the quantum of thermal conductivity appearing at the very beginning. There are emphasized the similarity and differences in the construction of the theory of electron conductivity and the theory of heat conduction

Keywords: nanophysics, nanoelectronics, phonon transport, thermal conductivity quantum, transmission coefficient, phonon modes, Debye model, phonon scattering

\section{1. Введение}

При транспорте электронов переносится как заряд, так и тепло. В металлах почти все тепло переносится электронами, а в полупроводниках только меньшая часть тепла переносится электронами, и почти все тепло переносится фононами.

Поток тепла, вызванный транспортом фононов вдоль проводника (ось $x$ )

$$
J_{Q x}^{p h}=-\kappa_{L} \frac{d T}{d x},\left[B m / M^{2}\right]
$$

где коэффициентом пропорциональности потока тепла градиенту температуры является удельная решеточная теплопроводность $\kappa_{L}$. У алмаза, одного из лучших проводников тепла, $\kappa_{L} \approx 2000 \mathrm{Bm} / \mathrm{M} \cdot \mathrm{K}, \mathrm{y}$ такого плохого проводника тепла как стекло $\kappa_{L} \approx 1$
$B m / \mu \cdot K$, а у воздуха $\kappa_{L} \approx 0.025 \mathrm{Bm} / \mathrm{\mu} \cdot \mathrm{K}$. Если электрическая проводимость твердых материалов меняется на 20 и больше порядков, то их решеточная теплопроводность изменяется всего на 3-4 порядка. Тем не менее, есть много общего в описании переноса тепла электронами и фононами в рамках транспортной модели Ландауэра - Датты Лундстрома (ЛДЛ) [1-4]. Далее упомянем лишь необходимые нам сведения о фононах.

\section{2. Обзор элементарной физики фононов}

Физика фононов подробно изложена в [5-8]. Характерный ход дисперсии $E(k)$ для электронов качественно показан на рис. 1 , а дисперсии $\hbar \omega(q)$ для фононов - на рис. 2. 


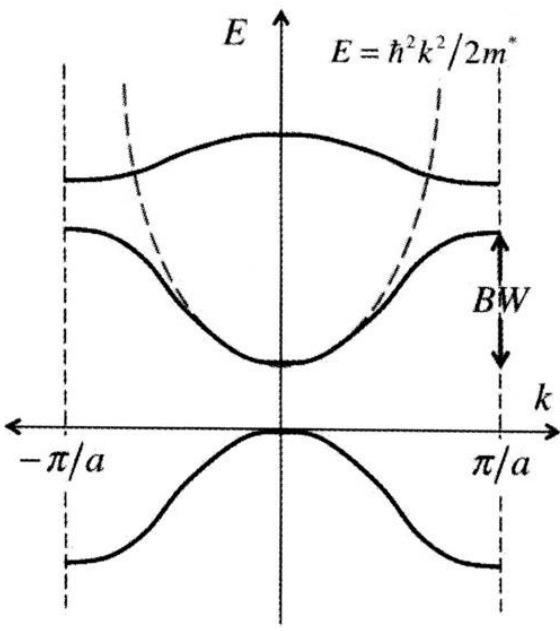

Рис. 1. Упрощенная зонная структура. Ширина зоны проводимости помечена BW/Band Width. Часто используемая параболическая дисперсия показана пунктиром

Кристаллическая решетка периодична в реальном пространстве, а дисперсия - в $k$-пространстве. Аналогично для фононов: только в $q$-пространстве. В обоих случаях решения реализуются в зоне Бриллюэна и ширины обоих зон конечны.

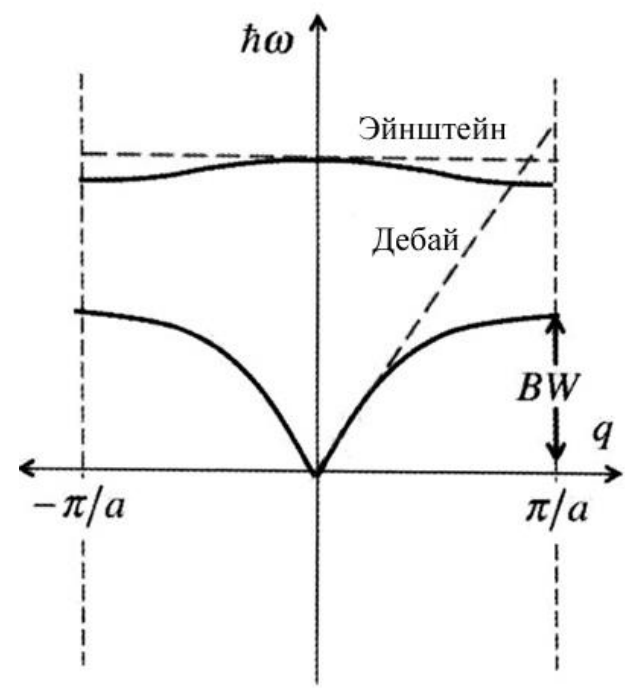

Рис. 2. Упрощенный ход дисперсии для фононов. Пунктиром показаны простейшие модели Эйнштейна и Дебая

Подробнее ход дисперсии для фононов показан на рис. 3.

Для кристаллических 3D проводников при распространении колебаний по решетке возможны три состояния поляризации: одно продольное, в направлении распространения колебаний (longitudinal/L) и два поперечных (transverse/T). В области низких энергий фононные моды называют акустическими/А. Вблизи нулевых волновых чисел $(q \rightarrow 0)$ у акустических мод дисперсия линейная. Для продольной акустической моды LA

$$
\begin{aligned}
& \omega(q)=v_{s} q, \\
& v_{s}=\sqrt{k_{\text {elas }} / m_{\text {eff }}},
\end{aligned}
$$

где скорость звука $v_{s}$ определяется упругой постоянной $k_{\text {elas }}$ и массой $m_{\text {eff }}$. Характерные скорости звука порядка $5 \times 10^{3} \mathrm{M} / \mathrm{c}$, что примерно в 20 раз меньше скорости электронов. Из уравнений (2) следует, что у веществ с тяжелыми атомами скорость звука меньше и соответственно меньше ширина фононной зоны дисперсии по сравнению с веществами из легких атомов.

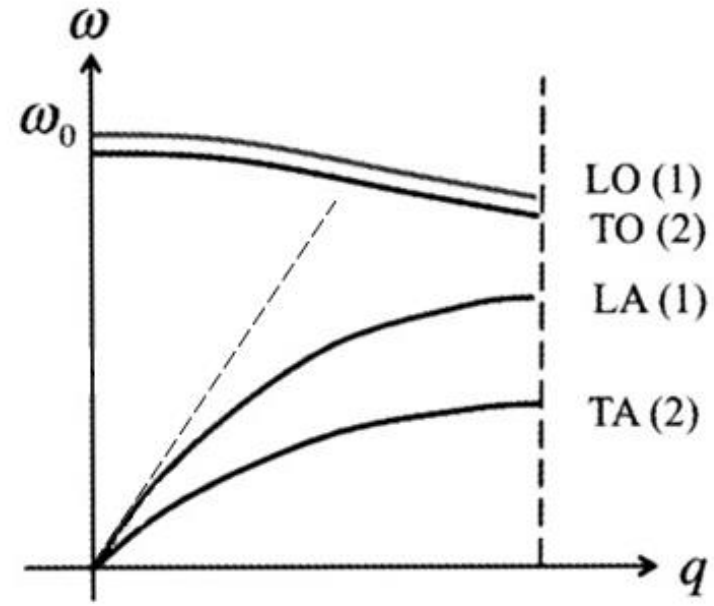

Рис. 3. Дисперсионные кривые для фононов

На рис. 3 показаны также три оптические моды. Дисперсия этих мод незначительна: частота оптических мод $L$ и $T$ сравнительно мало зависит от $q$. Различие между акустическими и оптическими модами состоит в том, что в области малых $q \approx 0$ соседние атомы смещаются в одном и том же направлении для акустических мод и в противоположных направлениях для оптических мод. В полярных веществах свет взаимодействует с оптическими модами; отсюда и их название. Скорость фононов определяется наклоном зависимости частоты от смещения $\omega(q)$ : акустические моды характеризуются относительной большей скоростью фононов по сравнению с оптическими модами. Поэтому можно ожидать, что именно акустические моды переносят большую часть тепла.

В качестве реалистического примера приводим фононный спектр графена (рис. 4), построенный на основе экспериментальных данных, хорошо согласующихся с результатами расчетов методами теории функционала плотности [9-11].

В элементарной ячейке графена два атома углерода, что обусловливает наличие в спектре дисперсии графена шести фононных ветвей - трех оптических (LO, TO, ZO) и трех акустических (LA, TA, ZA). Последние связаны со смещением всей ячейки из положения равновесия, а оптическим модам отвечают сдвиги атомов в самой элементарной ячейке с сохранением положения центра масс. Фононные моды LA и TA соответствуют продольным и поперечным колебаниям атомов 
углерода в плоскости графена, а мода ZA (flexural mode) - колебаниям атомов углерода в направлении, перпендикулярном направлению колебаний мод LA и ТА, с выходом атомов из плоскости графена. Моды LA и TA характеризуются линейной дисперсией; соответствующие этим модам скорости звука составляют 21300 и $13600 \mathrm{M} / \mathrm{c}$, соответственно. Все теплофизические свойства графена (тепло проводность, теплоемкость и др.) определяются фононами.

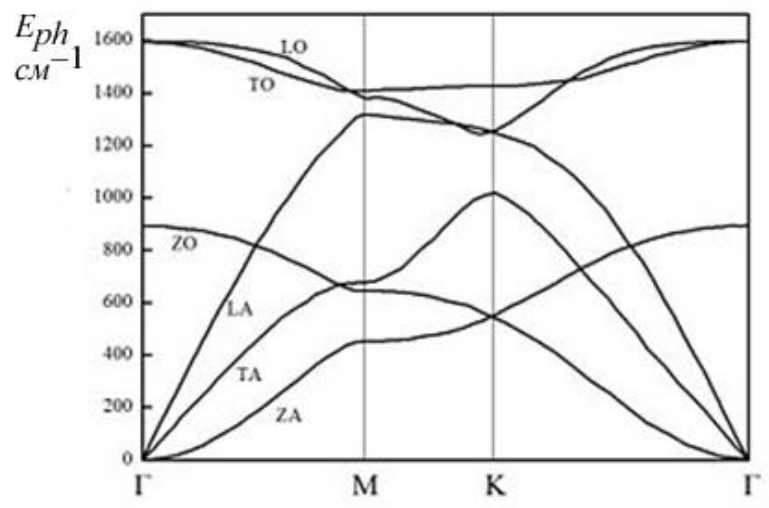

Рис. 4. Фононный спектр графена, опирающийся на совокупные экспериментальные и расчетные данные

3. Обобщенная модель Ландауэра-ДаттыЛундстрома переноса тепла фононами

Электрический ток в обобщенной транспортной модели ЛДЛ $[3,4,12]$

$$
I=\frac{2 q}{h} \int T_{e l}(E) M_{e l}(E)\left(f_{1}-f_{2}\right) d E[A]
$$

пропорционален произведению коэффициента прохождения $T_{e l}(E)$ или иначе вероятности прохождения проводника по каналу проводимости энергии $E$, числу мод проводимости $M_{e l}(E)$ при этой энергии и разности фермиевских функций на контактах проводника.

Для описания транспорта фононов нам необходимо уравнение, аналогичное (3). Как и в случае электронов, контакты на концах проводника достаточно массивны настолько, чтобы поддерживать тепловое равновесие фононов при разных температурах контактов. Если в случае электронов состояния контактов заселяются в соответствии со статистикой Ферми-Дирака, то в случае фононов - в соответствии со статистикой Бозе-Эйнштейна

$$
n_{0}(\hbar \omega)=\frac{1}{e^{h \omega / k T}-1} .
$$

Пусть температура на левом и правом контактах будет соответственно $T_{1}$ и $T_{2}$. Как и в случае электронов, предполагается, что контакты идеальные, другими словами, отражение фононов на контактах не происходит, и коэффициент прохождения действительно соответствует прохождению фононов через весь канал проводимости.

Теперь легко переписать выражение (3) применительно к фононам. Для потока фононов энергию электронов $E$ заменим на энергию фононов $\hbar \omega$. В случае электронов переносятся заряды $q$, а в случае фононов - кванты энергии $\hbar \omega$ : заменим $q$ на $\hbar \omega$ и внесем $\hbar \omega$ под знак интеграла. Двойка в уравнении (3) для тока электронов соответствует вырождению по спину. Исключим ее из уравнения для фононов, а число состояний поляризации фононов включим в число фононных мод $M_{p h}(\hbar \omega)$. Окончательно для тепла, переносимого фононами, имеем:

$$
Q=\frac{1}{h} \int(\hbar \omega) T_{p h}(\hbar \omega) M_{p h}(\hbar \omega)\left(n_{1}-n_{2}\right) d(\hbar \omega)[B m] .
$$

Как и в случае электронов, нас интересует режим линейного отклика, или иначе квазиравновесного транспорта. В этом режиме $T_{1} \approx T_{2}$ и $n_{0} \approx n_{1} \approx n_{2} \approx n_{0}$, что позволяет разложить $n_{2}$ относительно $n_{1}$ в ряд Тейлора и ограничиться первым порядком:

$$
n_{2} \approx n_{1}+\frac{\partial n_{0}}{\partial T} \Delta T,
$$

так что нужная нам разность

$$
n_{1}-n_{2} \approx-\frac{\partial n_{0}}{\partial T} \Delta T
$$

Производную $\partial n_{0} / \partial T$ находим дифференцированием бозевского распределения (4):

$$
\frac{\partial n_{0}}{\partial T}=\frac{\hbar \omega}{T}\left(-\frac{\partial n_{0}}{\partial(\hbar \omega)}\right)
$$

где

$$
\frac{\partial n_{0}}{\partial(\hbar \omega)}=\left(-\frac{1}{k T}\right) \frac{e^{\hbar \omega / k T}}{\left(e^{h \omega / k T}-1\right)^{2}} .
$$

Теперь уравнение (5) для малой разности температур на контактах можно переписать следующим образом:

$$
Q=-K_{L} \Delta T,
$$

где решеточная теплопроводность

$K_{L}=\frac{k^{2} T}{h} \int T_{p h}(\hbar \omega) M_{p h}(\hbar \omega)\left[\left(\frac{\hbar \omega}{k T}\right)^{2}\left(-\frac{\partial n_{0}}{\partial(\hbar \omega)}\right)\right] d(\hbar \omega)[B m / K]$.

Уравнение (10) есть закон теплопроводности Фурье, согласно которому поток тепла антинаправлен градиенту температуры. Полезно обратить внимание на следующую схожесть теплопроводности (11) и электронной проводимости

$$
G=\frac{2 q^{2}}{h} \int T_{e l}(E) M_{e l}(E)\left(-\frac{\partial f_{0}}{\partial E}\right) d E .
$$

Производная

$$
W_{e l}(E)=\left(-\frac{\partial f_{0}}{\partial E}\right)
$$

известна как фермиевское окно проводимости, выделяющее те моды проводника, которые только и 
могут внести вклад в электронную проводимость. Окно проводимости нормировано на единицу:

$$
\int_{-\infty}^{+\infty}\left(-\frac{\partial f_{0}}{\partial E}\right) d E=1
$$

Аналогичную роль окна проводимости для фононов играет следующее выражение:

$$
W_{p h}(\hbar \omega)=\frac{3}{\pi^{2}}\left(\frac{\hbar \omega}{k T}\right)\left(\frac{\partial n_{0}}{\partial(\hbar \omega)}\right),
$$

в котором численный коэффициент необходим для нормировки на единицу выражения в квадратных скобках уравнения (11):

$$
\int_{0}^{+\infty}\left(\frac{\hbar \omega}{k T}\right)^{2}\left(-\frac{\partial n_{0}}{\partial(\hbar \omega)}\right) d(\hbar \omega)=\frac{\pi^{2}}{3},
$$

так что окончательно решеточная теплопроводность

$$
K_{L}=\frac{\pi^{2} k^{2} T}{3 h} \int T_{p h}(\hbar \omega) M_{p h}(\hbar \omega) W_{p h}(\hbar \omega) d(\hbar \omega) .
$$

В этом выражении численный коэффициент

$$
g_{0} \equiv \pi^{2} k^{2} T / 3 h \approx\left(9.456 \times 10^{-13} \mathrm{Bm} / \mathrm{K}^{2}\right) T,
$$

который мы обсудим позже, есть квант теплопроводности, впервые подтвержденный экспериментально в 2000 году [13].

Сравнивая выражения для решеточной теплопроводности (17) и электронной проводимости (12), опять убеждается в их схожести: и та и другая проводимости пропорциональны соответствующему кванту проводимости, умноженному на интеграл от произведения трех величин - коэффициента прохождения, числа мод и окна проводимости.

Профили окон проводимости для электронов и фононов показаны на рис. 5, 6.

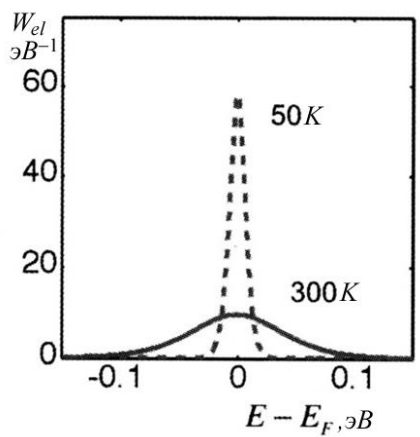

Рис. 5. Окно проводимости для электронов (13) при двух разных температурах. Энергия $E$ может быть как больше фермиевской, так и меньше ее

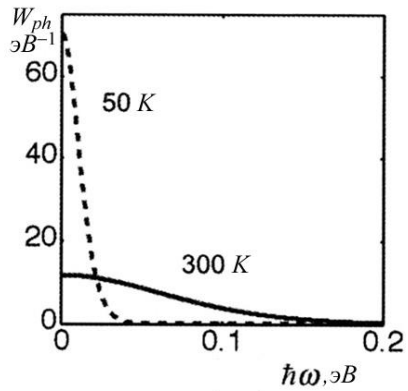

Рис. 6. Окно проводимости для фононов (15) при двух разных температурах. Энергия фононов может быть только положительной
Окна проводимости для электронов и фононов не только схожи, но и имеют приблизительно одинаковую ширину в $\approx \pm 2 k T$.

\section{4. Теплопроводность массивных провод-} ников

Теплопроводность диффузионных проводников является их ключевым свойством, определяющим работоспособность разнообразных электронных устройств. По аналогии с транспортом электронов (ф-ла (86) из [12]) коэффициент прохождения для фононов

$$
T_{p h}(\hbar \omega)=\left.\frac{\lambda_{p h}(\hbar \omega)}{\lambda_{p h}(\hbar \omega)+L}\right|_{L \gg \lambda_{p h}} \rightarrow \frac{\lambda_{p h}(\hbar \omega)}{L},
$$

где $L$ - длина 3D проводника, а $\lambda_{p h}(\hbar \omega)$ есть длина свободного пробега фонона частоты $\omega$. Кроме того, число фононных мод, очевидно, пропорционально площади поперечного сечения 3D проводника:

$$
M_{p h}(\hbar \omega) \propto A .
$$

Умножая и деля уравнение (10) на $A / L$, получаем

$$
Q=-\left(K_{L} \frac{L}{A}\right) A \frac{\Delta T}{L},
$$

откуда следует постулированное в самом начале уравнение (1) для потока тепла:

$$
\frac{Q}{A} \equiv J_{Q x}^{p h}=-\kappa_{L} \frac{d T}{d x}
$$

с удельной решеточной теплопроводностью

$$
\kappa_{L}=K_{L} \frac{L}{A}
$$

или после подстановки (19) в (17) в развернутом виде окончательно получаем

$$
\kappa_{L}=\frac{\pi^{2} k^{2} T}{3 h} \int \frac{M_{p h}(\hbar \omega)}{A} \lambda_{p h}(\hbar \omega) W_{p h}(\hbar \omega) d(\hbar \omega) .
$$

Поскольку именно окно проводимости фононов $W_{p h}(\hbar \omega)$ определяет какие именно фононные моды могут принимать участие в проводимости тепла, естественно ввести среднее число активных фононных мод на единицу площади поперечного сечения проводника

$$
\left\langle M_{p h} / A\right\rangle \equiv \int \frac{M_{p h}(\hbar \omega)}{A} W_{p h}(\hbar \omega) d(\hbar \omega),
$$

так что в компактном виде

$$
\kappa_{L}=\frac{\pi^{2} k^{2} T}{3 h}\left\langle M_{p h} / A\right\rangle\left\langle\left\langle\lambda_{p h}\right\rangle\right\rangle,
$$

где усредненное значение длины свободного пробега фононов 


$$
\left\langle\left\langle\lambda_{p h}\right\rangle\right\rangle=\frac{\int \frac{M_{p h}(\hbar \omega)}{A} \lambda_{p h}(\hbar \omega) W_{p h}(\hbar \omega) d(\hbar \omega)}{\int \frac{M_{p h}(\hbar \omega)}{A} W_{p h}(\hbar \omega) d(\hbar \omega)} .
$$

Итак, паре фононных транспортных уравнений (22) и (26) соответствует схожая по форме пара электронных транспортных уравнений $[3,4,12]$ :

$$
\begin{gathered}
J_{x}=\frac{\sigma}{q} \frac{d\left(E_{F}\right)}{d x}, \\
\sigma=\frac{2 q^{2}}{h}\left\langle M_{e l} / A\right\rangle\left\langle\left\langle\lambda_{e l}\right\rangle\right\rangle .
\end{gathered}
$$

Градиент электрохимического потенциала вызывает поток электронов, а градиент температуры - поток тепла. Термоэлектрические коэффициенты (26) и (29) имеют одинаковую структуру. Это всегда произведение соответствующего кванта прово-димости на число мод проводимости, участвующих в транспорте, и на усредненное значение средней длины свободного пробега соответствующего носи-теля (тока или тепла). К оценке этих трех величин для фононов мы вернемся позже.

\section{5. Удельная теплоемкость}

Хорошо известна пропорциональность между удельной теплопроводностью и удельной теплоемкостью при постоянном объеме $C_{V}$ [5-8]. Установим эту связь и покажем, что коэффициент пропорциональности между ними определяется усредненной должным образом средней длиной свободного пробега фононов $\left\langle\left\langle\Lambda_{p h}\right\rangle\right\rangle$ и средней скоростью фононов $\left\langle v_{p h}\right\rangle$, а именно:

$$
\kappa_{L}=\frac{1}{3}\left\langle\left\langle\Lambda_{p h}\right\rangle\right\rangle\left\langle v_{p h}\right\rangle C_{V} .
$$

Энергия фононов на единицу объема, очевидно, дается выражением:

$$
E_{p h}=\int_{0}^{\infty}(\hbar \omega) D_{p h}(\hbar \omega) n_{0}(\hbar \omega) d(\hbar \omega),
$$

где $D_{p h}(\hbar \omega)$ есть плотность фононных состояний. По определению,

$$
\begin{aligned}
& C_{V} \equiv \frac{\partial E_{p h}}{\partial T}=\frac{\partial}{\partial T} \int_{0}^{\infty}(\hbar \omega) D_{p h}(\hbar \omega) n_{0}(\hbar \omega) d(\hbar \omega)= \\
& =\int_{0}^{\infty}(\hbar \omega) D_{p h}(\hbar \omega)\left(\frac{\partial n_{0}(\hbar \omega)}{\partial T}\right) d(\hbar \omega)= \\
& =\frac{\pi^{2} k^{2} T}{3} \int_{0}^{\infty} D_{p h}(\hbar \omega) W_{p h}(\hbar \omega) d(\hbar \omega),
\end{aligned}
$$

где использовались уравнения (8) и (15).

Умножение и деление коэффициента теплопроводности (24) на теплоемкость (32) дает искомую пропорциональность:

$$
\kappa_{L}=\left[\frac{\frac{1}{h} \int_{0}^{\infty} \frac{M_{p h}(\hbar \omega)}{A} \lambda_{p h}(\hbar \omega) W_{p h}(\hbar \omega) d(\hbar \omega)}{\int_{0}^{\infty} D_{p h}(\hbar \omega) W_{p h}(\hbar \omega) d(\hbar \omega)}\right] C_{V} .
$$

Для получения окончательного результата в виде уравнения (30) и корректной интерпретации коэффициента пропорциональности между $\kappa_{L}$ и $C_{V}$ нужно вернуться к связи между средней длиной свободного пробега и коэффициентом прохождения (19), имеющей одинаковый вид как для электронов, так и для фононов.

\section{6. Средняя длина свободного пробега и} коэффициент прохождения

Связь этих двух важнейших характеристик диффузионного транспорта проще всего проиллюстрировать на примере электронного транспорта по 1D проводнику в диффузионном режиме (рис. 7).

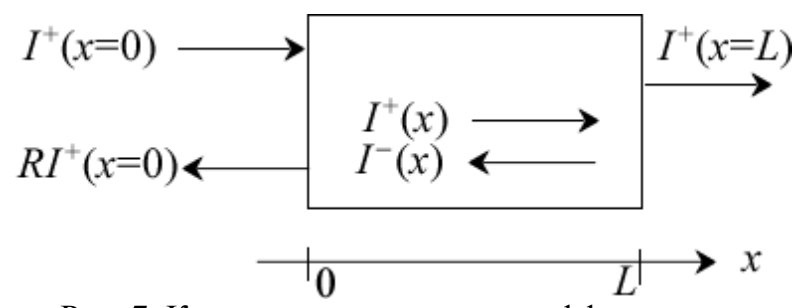

Рис. 7. К выводу связи между коэффициентом прохождения и средней длиной свободного пробега на примере однородного 1D проводника

Рассмотрим однородный $1 \mathrm{D}$ проводник в диффузионном режиме. Левый контакт впрыскивает электроны с током $I^{+}(x=0)$. Доля электронов $T$ войдет в правый контакт с током $I^{+}(x=L)=T I^{+}(x=0)$. Оставшаяся доля вернется в левый контакт (рассеяние назад) с током $I^{-}(x=0)=R I^{+}(x=0) . \quad$ В отсутствии процессов рекомбинации $T+R=1$. Предполагается также, что правый контакт идеальный, поглощает все входящие в него электроны. Результирующий ток, очевидно, будет $I=(1-R) I^{+}(0)=T I^{+}(0)$. Так или иначе, в проводнике имеют место как прямые, так и обратные потоки электронов, и нам необходимо описать их пространственное распределение.

Определим обратное значение средней длины свободного пробега $1 / \lambda$ как вероятность (на единицу длины) обращения положительного потока электронов в отрицательный и наоборот. Именно исходя из этого определения, величину $\lambda$ и называют иногда средней длиной свободного обратного рассеяния (mean-free-pass for backscattering) или проще средней длиной свободного пробега. В диффузионном проводнике некоторая доля положительного потока в результате рассеяния назад обращается в отрицательный. В проводнике формируется отрицательный поток и его некоторая доля в результате рассеяния назад обращается, усиливая положительный поток. В результате градиент положительного тока складывается из двух величин: 


$$
\frac{d I^{+}(x)}{d x}=-\frac{I^{+}(x)}{\lambda}+\frac{I^{-}(x)}{\lambda} .
$$

Пренебрегая процессами рекомбинации, результирующий ток

$$
I=I^{+}(x)-I^{-}(x)
$$

является постоянной величиной, так что градиент тока

$$
\frac{d I^{+}(x)}{d x}=-\frac{I}{\lambda}
$$

является константой. Другими словами, ток спадает линейно вдоль проводника:

$$
I^{+}(x)=I^{+}(0)-\frac{I}{\lambda} x .
$$

Воспользуемся полученным уравнением для вычисления тока, входящего в правый контакт:

$$
\begin{aligned}
& I^{+}(L)=I^{+}(0)-\frac{I}{\lambda} L=I^{+}(0)-\left[I^{+}(L)-I^{-}(L)\right] \frac{L}{\lambda}= \\
& =I^{+}(0)-I^{+}(L) \frac{L}{\lambda}
\end{aligned}
$$

где мы воспользовались уравнением баланса (35) и тем, что правый контакт идеальный $\left(I^{-}(L)=0\right)$. Из последнего равенства находим

$$
I^{+}(L)=\frac{\lambda}{\lambda+L} I^{+}(0)=T I^{+}(0),
$$

если провести аналогичные рассуждения для впрыскивания электронов правым контактом, то получим аналогичное уравнение для электронов, входящих в левый контакт, а именно: $I^{-}(0)=T^{\prime} I^{-}(L)$. Для однородного проводника $T^{\prime}=T$. Проводник под напряжением не является однородным, но нас интересует режим линейного отклика, так что вполне приемлемо положить $T^{\prime} \approx T$. Окончательно, в предположении независимости друг от друга мод проводимости получаем искомое уравнение (19), связывающее коэффициент прохождения со средней длиной свободного пробега:

$$
T(E)=\frac{\lambda(E)}{\lambda(E)+L} .
$$

Вывод уравнения (40) сделан в рамках простой модели, что никак не мешает успешному и широкому использованию его на практике. Важным моментом в проведенных рассуждениях является интерпретация $1 / \lambda$ как вероятности (на единицу длины) обращения потока частиц в обратном направлении в результате рассеяния. Именно поэтому саму длину $\lambda$, как уже упоминалось, часто называют средней длиной свободного рассеяния назад.

Установим связь между $\lambda$ и временем рассеяния $\tau$. Пусть электрон совершает акт рассеяния в изотропном 1D проводнике. У него есть две возможности: рассеяться вперед и рассеяться назад. Только рассеяние назад существенно для определения средней длины свободного пробега. Отсюда следует, что средняя длина рассеяния назад равна удвоенному значению средней длины рассеяния:

$$
\lambda_{1 D}(E)=2 \Lambda(E)=2 v(E) \tau(E) .
$$

Для проводника произвольной размерности средняя длина рассеяния назад дается выражением [14]

$$
\lambda(E)=2 \frac{\left\langle v_{x}^{2} \tau\right\rangle}{\left\langle\left|v_{x}\right|\right\rangle},
$$

где усреднение для 2D и $3 \mathrm{D}$ проводников ведется по углам. Для изотропных проводников

$$
\begin{aligned}
& \lambda_{2 D}(E)=\frac{\pi}{2} v(E) \tau(E), \\
& \lambda_{3 D}(E)=\frac{4}{3} v(E) \tau(E) .
\end{aligned}
$$

Для времени рассеяния $\tau$ часто используется степенной закон

$$
\tau(E)=\tau_{0}\left(\frac{E-E_{C}}{k T}\right)^{s},
$$

где значение показателя степени отражает тот или иной механизм рассеяния. Для акустических фононов в 3D проводниках с параболической дисперсией $s=-1 / 2$, для рассеяния на ионизированных примесях $s=+3 / 2$ [15].

Аналогичный степенной закон часто используется для средней длины рассеяния:

$$
\lambda(E)=\lambda_{0}\left(\frac{E-E_{C}}{k T}\right)^{r} .
$$

Для параболической зонной структуры $v(E) \propto E^{1 / 2}$, так что $r=s+1 / 2$. Для акустических фононов, соответственно, $r=0$, а для рассеяния на ионизированных примесях $r=2$.

Возвращаясь к нашей первоначальной задаче выводу уравнения (30) из (33), для 3D проводника, согласно (44), имеем:

$$
\lambda_{p h}(\hbar \omega)=\frac{4}{3} v_{p h}(\hbar \omega) \tau_{p h}(\hbar \omega),
$$

где, согласно (41),

$$
v_{p h}(\hbar \omega) \tau_{p h}(\hbar \omega)=\Lambda_{p h}(\hbar \omega),
$$

так что окончательно

$$
\lambda_{p h}(\hbar \omega)=\frac{4}{3} \Lambda_{p h}(\hbar \omega) .
$$

\section{7. Плотность фононных состояний}

\section{фононные моды}

Ранее было показано, что для 3D проводника число мод и плотность электронных состояний связаны соотношением (ф-ла (63) в [12]):

$$
M_{e l}(E)=A M_{3 D}(E)=A \frac{h}{4}\left\langle v_{x}^{+}(E)\right\rangle D_{3 D}(E) .
$$


Мы хотим эту формулу переписать применительно к фононам. Сначала вспомним, что спиновое вырождение $g_{S}=2$ входит в плотность состояний, так что в пересчете на один спин

$$
D_{3 D}(E)=2 D_{3 D}^{\prime}(E) .
$$

Далее, для сферических зон в 3D

$$
\left\langle v_{x}^{+}(E)\right\rangle=\frac{v_{e l}(E)}{2} .
$$

Сочетая (51) и (52) с (50) и переходя к фононам, получаем

$$
\begin{aligned}
& M_{p h}(\hbar \omega)=A \frac{h}{2}\left(\frac{v_{p h}(\hbar \omega)}{2}\right) 2 D_{p h}(\hbar \omega)= \\
& =A \frac{h}{4} v_{p h}(\hbar \omega) D_{p h}(\hbar \omega)
\end{aligned}
$$

Подставляя (49) и (53) в (33), получаем очередной промежуточный результат:

$$
\kappa_{L}=\left[\frac{\frac{1}{3} \int_{0}^{\infty} \Lambda_{p h}(\hbar \omega) v_{p h}(\hbar \omega) D_{p h}(\hbar \omega) W_{p h}(\hbar \omega) d(\hbar \omega)}{\int_{0}^{\infty} D_{p h}(\hbar \omega) W_{p h}(\hbar \omega) d(\hbar \omega)}\right] C_{V} .
$$

Умножая и деля (54) на

$$
\int_{0}^{\infty} v_{p h}(\hbar \omega) D_{p h}(\hbar \omega) W_{p h}(\hbar \omega) d(\hbar \omega),
$$

окончательно получаем искомое уравнение (30) с коэффициентом пропорциональности между $\kappa_{L}$ и $C_{V}$ в виде произведения средней длины свободного пробега фононов

$$
\left\langle\left\langle\Lambda_{p h}\right\rangle\right\rangle \equiv \frac{\int_{0}^{\infty} \Lambda_{p h}(\hbar \omega) v_{p h}(\hbar \omega) D_{p h}(\hbar \omega) W_{p h}(\hbar \omega) d(\hbar \omega)}{\int_{0}^{\infty} v_{p h}(\hbar \omega) D_{p h}(\hbar \omega) W_{p h}(\hbar \omega) d(\hbar \omega)}
$$

и средней скорости фононов

$$
\left\langle v_{p h}\right\rangle \equiv \frac{\int_{0}^{\infty} v_{p h}(\hbar \omega) D_{p h}(\hbar \omega) W_{p h}(\hbar \omega) d(\hbar \omega)}{\int_{0}^{\infty} D_{p h}(\hbar \omega) W_{p h}(\hbar \omega) d(\hbar \omega)},
$$

усредненных должным образом.

Уравнение (30) часто используется для вычисления средней длины сводного пробега фононов из результатов измерений теплопроводности и теплоемкости. Для этого нужно знать среднюю скорость фононов, которая нередко предполагается равной продольной составляющей скорости звука. Приведенные выше выкладки точно определяют смысл усреднений при вычислении средней длины свободного пробега фононов и их средней скорости. Например, задавшись дисперсией фононов всегда можно вычислить их среднюю скорость по (57). Как правило, вычисленная таким образом скорость сильно отличается от продольной составляющей скорости звука, что далее ведет к ошибочным результатам по средней длине свободного пробега фононов [16].

Рассмотрим плотность фононных состояний и число фононных мод с более общих позиций. Плотность состояний фононов определяется аналогично плотности состояний электронов, как число разрешенных фононных состояний на единицу энергии и единицу длины $L$ для $1 \mathrm{D}$ проводников, площади поперечного сечения $A$ для 2D проводников и на единицу объема $\Omega=L \cdot A$ для $3 \mathrm{D}$ проводников. В случае электронов число разрешенных состояний со значением импульса меньшим, чем заданное значение $p$, для проводников размерности $d=\{1,2,3\}$ [17]

$$
N(p)=\left\{2 \frac{L}{h / p}, \quad \pi \frac{L \cdot W}{(h / p)^{2}}, \quad \frac{4 \pi}{3} \frac{L \cdot A}{(h / p)^{3}}\right\} .
$$

Аналогично, число разрешенных фононных состояний со значением волнового вектора $k=p / \hbar$ меньшим, чем заданное значение $k$,

$$
\begin{aligned}
& N_{1 D}(k)=\frac{2 k}{2 \pi / L}, \\
& N_{2 D}(k)=\frac{\pi k^{2}}{4 \pi^{2} / L^{2}}, \\
& N_{3 D}(k)=\frac{4 \pi k^{3} / 3}{8 \pi^{3} / L^{3}},
\end{aligned}
$$

где единообразия ради далее удобно площадь 2D проводника обозначить как $L^{2} \equiv L \cdot W$, а объем для $3 \mathrm{D}$ проводника как $L^{3} \equiv \Omega=L \cdot A$. Величину $L^{d}$ условно назовем «объемом» проводника размерности $d$.

Плотность фононных состояний, согласно определению выше, очевидно, такова:

$$
\begin{aligned}
& D_{1 D}(k)=\frac{1}{L} \frac{d N_{1 D}(k)}{d k}=\frac{1}{\pi}, \\
& D_{2 D}(k)=\frac{1}{L^{2}} \frac{d N_{2 D}(k)}{d k}=\frac{k}{2 \pi}, \\
& D_{3 D}(k)=\frac{1}{L^{3}} \frac{d N_{3 D}(k)}{d k}=\frac{k^{2}}{2 \pi^{2}} .
\end{aligned}
$$

Плотность фононных состояний часто выражают через групповую скорость фононов

а именно [18]:

$$
v_{g}(\omega)=d \omega / d k,
$$

$$
\begin{aligned}
& D_{1 D}(\omega)=\frac{1}{L} \frac{d N_{1 D}(k)}{d \omega}=\frac{1}{L} \frac{d N_{1 D}(k)}{d k} \frac{d k}{d \omega}=\frac{1}{\pi v_{g}(\omega)}, \\
& D_{2 D}(\omega)=\frac{1}{L^{2}} \frac{d N_{2 D}(k)}{d \omega}=\frac{1}{L^{2}} \frac{d N_{2 D}(k)}{d k} \frac{d k}{d \omega}=\frac{k(\omega)}{2 \pi v_{g}(\omega)}, \\
& D_{3 D}(\omega)=\frac{1}{L^{3}} \frac{d N_{3 D}(k)}{d \omega}=\frac{1}{L^{3}} \frac{d N_{3 D}(k)}{d k} \frac{d k}{d \omega}=\frac{k^{2}(\omega)}{2 \pi^{2} v_{g}(\omega)} .
\end{aligned}
$$


Количество тепла, переносимого фононами, определяется выражением (5), в котором число состояний поляризации фононов учтено в числе фононных мод $M_{p h}(\hbar \omega)$. Перепишем это выражение через интеграл по частоте:

$$
Q=\frac{1}{2 \pi} \int_{0}^{\infty}(\hbar \omega) T_{p h}(\omega) M_{p h}(\omega)\left(n_{1}-n_{2}\right) d \omega .
$$

При переносе тепла по 1D проводнику $M_{p h}(\omega)$ есть просто единица, по 2D проводнику $M_{p h}(\omega)$ пропорционально ширине проводника, а по 3D проводнику - площади его поперечного сечения:

$$
M_{p h}(\omega)=\left\{M_{1 D}(\omega), W M_{2 D}(\omega), A M_{3 D}(\omega)\right\} .
$$

Для вычисления и интерпретации $M_{2 D}(\omega)$ и $M_{3 D}(\omega)$ нам потребуются соответствующие выражения для потоков тепла

$$
\begin{aligned}
& J_{Q x}^{p h}=\left\{J_{Q}^{p h}(1 D), J_{Q}^{p h}(2 D), J_{Q}^{p h}(3 D)\right\}= \\
& =\{Q, Q / W, Q / A\},
\end{aligned}
$$

связанных непосредственно с количеством переносимого тепла (33), а именно [18]:

$$
\begin{aligned}
& J_{Q}^{p h}(1 D)=\int_{0}^{\infty}(\hbar \omega) T_{p h}(\omega)\left[\frac{1}{2} v_{g}(\omega) D_{1 D}(\omega)\right]\left(n_{1}-n_{2}\right) d \omega, \\
& J_{Q}^{p h}(2 D)=\int_{0}^{\infty}(\hbar \omega) T_{p h}(\omega)\left[\frac{1}{2} \frac{2 v_{g}(\omega)}{\pi} D_{2 D}(\omega)\right]\left(n_{1}-n_{2}\right) d \omega, \\
& J_{Q}^{p h}(3 D)=\int_{0}^{\infty}(\hbar \omega) T_{p h}(\omega)\left[\frac{1}{2} \frac{v_{g}(\omega)}{2} D_{3 D}(\omega)\right]\left(n_{1}-n_{2}\right) d \omega,
\end{aligned}
$$

где плотности фононных состояний определяются уравнениями (62). Уравнивая потоки тепла (66) с количеством перенесенного тепла (63) согласно (65) и учитывая выражения для плотности фононных состояний (62), окончательно получаем:

$$
\begin{aligned}
& M_{1 D}(\omega)=\pi\left[v_{g}(\omega)\right] D_{1 D}(\omega)=1, \\
& M_{2 D}(\omega)=\pi\left[\frac{2 v_{g}(\omega)}{\pi}\right] D_{2 D}(\omega)=\frac{k(\omega)}{\pi}, \\
& M_{3 D}(\omega)=\pi\left[\frac{v_{g}(\omega)}{2}\right] D_{3 D}(\omega)=\frac{k^{2}(\omega)}{4 \pi},
\end{aligned}
$$

или иначе число фононных мод (64), определяющих количество перенесенного тепла (63)

$$
M_{p h}(\omega)=\left\{\begin{array}{c}
M_{1 D}(\omega)=\pi\left[v_{g}(\omega)\right] \frac{1}{\pi v_{g}(\omega)}=1, \\
W M_{2 D}(\omega)=W \pi\left[\frac{2 v_{g}(\omega)}{\pi}\right] \frac{k(\omega)}{2 \pi v_{g}(\omega)}, \\
A M_{3 D}(\omega)=A \pi\left[\frac{v_{g}(\omega)}{2}\right] \frac{k^{2}(\omega)}{2 \pi^{2} v_{g}(\omega)},
\end{array}\right\}
$$

что позволяет интерпретировать число мод $M_{p h}(\omega)$ как максимальное число де-бройлевских полуволн, укладывающихся в «сечении» проводника, поперечном относительно направления движения фононов. Действительно, предполагая линейную зависимость $\omega(k)$, что вполне обосновано для LA и ТА акустических мод, имеем $k=2 \pi / \lambda$, так что уравнения (68) сводятся к

$$
M_{p h}(\omega)=\left\{\begin{array}{c}
M_{1 D}(\omega)=1, \\
W M_{2 D}(\omega)=W /(\lambda / 2), \\
A M_{3 D}(\omega)=A /\left[(4 / \pi)(\lambda / 2)^{2}\right]
\end{array}\right\},
$$

где множитель $4 / \pi$ появляется в связи с тем, что поперечное сечение 3D проводника предполагается круговым с площадью $A$, равной квадрату диаметра окружности, деленному на $4 / \pi$. Обратим также внимание на то, что выражения в квадратных скобках уравнений (68) есть ничто иное как усредненные по всем угловым переменным значения скорости фононов в направлении их движения (вдоль оси $+x$ в нашем рассмотрении) [19].

\section{8. Дебаевская модель}

В случае электронов ширина зоны проводимости $B W \gg k T$, так что заняты только состояния вблизи дна зоны проводимости и поэтому так неплохо работает модель эффективной массы с параболической дисперсией (рис. 1). Совсем другая ситуация в случае фононов: $B W \approx k T$ (рис. 4). Заняты состояния по всей зоне Бриллюэна. Акустические моды тем лучше аппроксимируются дебаевским линейным приближением (2), чем ближе волновые числа к центру зоны Бриллюэна (рис. 3 и 4).

Перепишем (2) в виде

$$
\hbar \omega=\hbar v_{D} q,
$$

где под дебаевской скоростью $v_{D}$ понимают среднюю скорость продольных и поперечных акустических мод. Тогда, по аналогии с электронами, плотность фононных состояний

$$
D_{p h}(\hbar \omega)=\frac{3(\hbar \omega)^{2}}{2 \pi^{2}\left(\hbar v_{D}\right)^{3}}\left[Д \varkappa^{-} \cdot M^{-3}\right],
$$

где множитель 3 учитывает три поляризации акустических мод. Обратим внимание, что в учебниках плотность фононных состояний чаще выражается в $\Gamma u^{-1} \cdot M^{-3}$ :

$$
D_{p h}(\hbar \omega)=\frac{D_{p h}(\omega)}{\hbar} .
$$

Число мод на единицу площади поперечного сечения проводника вычисляем по (53):

$$
M_{p h}(\hbar \omega)=\frac{3(\hbar \omega)^{2}}{4 \pi\left(\hbar v_{D}\right)^{2}} .
$$

Поскольку все состояния в зоне Бриллюэна при умеренных температурах стремятся быть заполненными, нужно проконтролировать, что действительно учтены все состояния. В кристалле с $N / \Omega$ атомами на единицу объема имеем $3 N / \Omega$ 
состояний на единицу объема. Полное число состояний дается интегралом

$$
\int_{0}^{\hbar \omega_{D}} D_{p h}(\hbar \omega) d(\hbar \omega),
$$

в котором верхний предел, определяемый дебаевской частотой, выбирается таким образом, чтобы получить правильное число состояний $3 N / \Omega$, а именно:

$$
\hbar \omega_{D}=\hbar v_{D}\left(\frac{6 \pi^{2} N}{\Omega}\right)^{1 / 3} \equiv k T_{D} .
$$

Дебаевская частота является предельной, выше которой фононные состояния уже не существуют или не учитываются. Это ограничение можно ввести и через дебаевский волновой вектор $q_{D}$ или через дебаевскую температуру $T_{D}$ через (74). Для $T \ll T_{D}$ заняты только состояния с $q \rightarrow 0$, для которых хорошо выполняется дебаевское приближение.

Теперь интегрирование в (24) можно вести только до дебаевской энергии:

$$
\kappa_{L}=\frac{\pi^{2} k^{2} T}{3 h} \int_{0}^{h \omega_{D}} \frac{M_{p h}(\hbar \omega)}{A} \lambda_{p h}(\hbar \omega) W_{p h}(\hbar \omega) d(\hbar \omega)
$$

и брать число фононных мод по (72). Интеграл можно взять численно или аналитически с приемлемым выражением для средней длины свободного пробега фононов. Именно таким образом была впервые вычислена решеточная теплопроводность [20, 21]. Дальнейшее развитие теория и методы вычисления термоэлектрических транспортных коэффициентов получили в работах [14, 16, 22].

\section{9. Рассеяние фононов}

Фононы рассеиваются на незаряженных дефектах, примесных атомах, изотопах, на других фононах, на поверхностях и границах, на электронах. Фонон-фононное рассеивание обязано тому, что потенциальная энергия колеблющегося атома в кристалле не совсем гармоническая. Все более высокие порядки учитываются в виде потенциала рассеяния. Рассматривают две возможности фононфононного взаимодействия. В обычном процессе два фонона взаимодействуя порождают третий фонон с сохранением импульса и энергии:

$$
\begin{aligned}
& \hbar \vec{q}_{1}+\hbar \vec{q}_{2}=\hbar \vec{q}_{3} \\
& \hbar \vec{w}_{1}+\hbar \vec{w}_{2}=\hbar \vec{w}_{3}
\end{aligned} .
$$

Поскольку суммарный импульс сохраняется, то такой процесс не может дать существенный вклад в перенос тепла.

B umklapp/U-процессах рассеяния суммарный импульс не сохраняется, генерируемый фонон характеризуется импульсом за пределами первой зоны Бриллюэна. Это может быть как ангармоничное фонон-фононное, так и электрон-фононное взаимодействия. U-рассеяние является основным процессом в переносе тепла, особенно при высоких темпе- ратурах. Ощутимый вклад дают также рассеяние на дефектах/D и на границах/B. Скорости всех трех процессов складываются, так что

$$
\frac{1}{\tau_{p h}(\hbar \omega)}=\frac{1}{\tau_{U}(\hbar \omega)}+\frac{1}{\tau_{D}(\hbar \omega)}+\frac{1}{\tau_{B}(\hbar \omega)}
$$

или через соответствующие длины свободного пробега

$$
\frac{1}{\lambda_{p h}(\hbar \omega)}=\frac{1}{\lambda_{U}(\hbar \omega)}+\frac{1}{\lambda_{D}(\hbar \omega)}+\frac{1}{\lambda_{B}(\hbar \omega)},
$$

поскольку для 3D сферических дисперсий $\lambda$ и $\tau$ связаны уравнением (47).

Для всех механизмов рассеяния предложены выражения для скоростей [23]. Например, при рассеянии на дефектах

$$
\frac{1}{\tau_{D}(\hbar \omega)} \propto \omega^{4}
$$

что напоминает рэлеевское рассеяние света на частицах пыли в атмосфере; высокочастотные фононы особенно чувствительны к рассеянию на малых дефектах. При рассеянии на границах и поверхностях

$$
\frac{1}{\tau_{B}(\hbar \omega)} \propto \frac{v_{p h}(\hbar \omega)}{L},
$$

где $L$ - наименьшая протяженность образца. Сложнее с $U$-рассеянием. Обычно используется выражение

$$
\frac{1}{\tau_{U}(\hbar \omega)} \propto T^{3} \omega^{2} e^{-T_{D} / b T}
$$

\section{0. Температурная зависимость тепло-} проводности

Температурная зависимость решеточной теплопроводности $\kappa_{L}$ кремния представлена на рис. 8 . Согласно уравнению (26), $\kappa_{L}$ пропорциональна числу фононных мод $\left\langle M_{p h} / A\right\rangle$ и среднему значению длины свободного пробега фононов $\left\langle\left\langle\lambda_{p h}\right\rangle\right\rangle$.

Изменение теплопроводности с температурой качественно можно объяснить следующим образом. Из определения числа мод (25) можно показать, что при низких температурах

$$
\left\langle M_{p h}\right\rangle \propto T^{3} ;(T \rightarrow 0)
$$

быстрое увеличение числа заселенных мод с температурой вызывает рост теплопроводности. Важную роль играет рассеяние на границах. По мере роста температуры все больше продуцируется коротковолновых фононов (большие значения $q$ ). Возрастает роль рассеяния таких фононов на дефектах. По мере приближения температуры к дебаевской заселяются все фононные моды и дальнейшее увеличение температуры уже не увеличивает числа мод. Более того, высокие температуры порождают $U$-процессы рассеяния и теплопроводность начинает падать. 


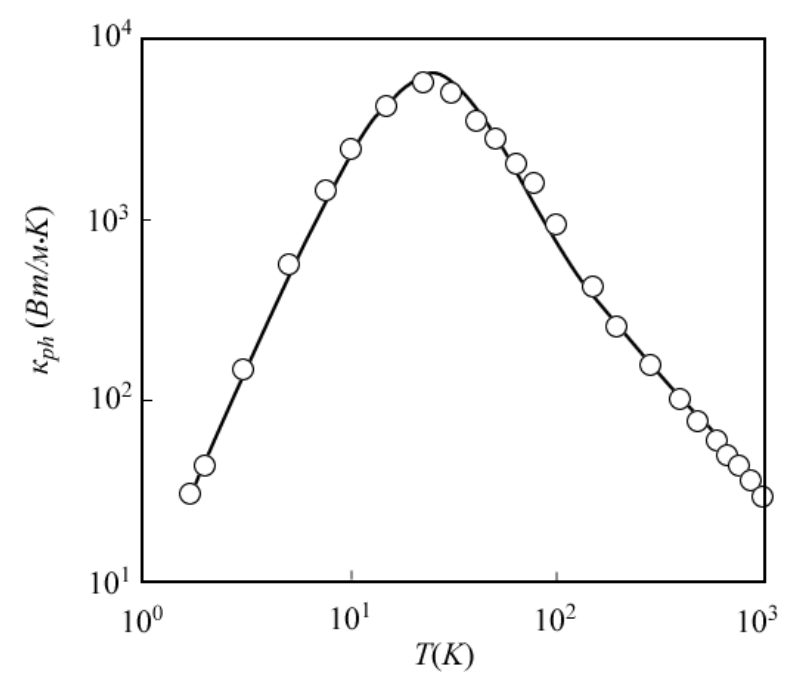

Рис. 8. Температурная зависимость решеточной теплопроводности кремния: экспериментальные точки по [24], расчетная кривая по [16]

11. Различие между решеточной теплопроводностью и электрической проводимостью

Уже подчеркивалось близкое подобие пары фононных транспортных уравнений (22) и (26) и пары электронных уравнений (28) и (29). Длины свободных пробегов электронов и фононов одного порядка. Почему же электрическая проводимость веществ меняется на много порядков, тогда как теплопроводность всего разнообразия веществ изменяется лишь на несколько порядков? Ответ кроется в различии между окнами проводимости для электронов (13) и фононов (15). Для тех и других рост температуры расширяет окна проводимости и тем самым увеличивает заселенность состояний. Для электронов, однако, все зависит от положения уровня Ферми, сдвигая который вверх или вниз можно менять проводимость на много порядков. Для фононов же ширина окна проводимости определяется только температурой.

Подчеркнем еще одно практически важное различие между описанием электронов и фононов, связанное с особенностями заселения состояний. В термоэлектрических устройствах $E_{F} \approx E_{C}$ и окна проводимости электронов и фононов схожи. У электронов, однако, ширина зон очень большая и лишь небольшое число состояний вблизи дна зоны проводимости заселено. Хорошо работает параболическая дисперсия и легко получить аналитические решения. В сопоставимой ситуации у фононов ширина зон незначительная, при повышенных температурах практически все состояния в зоне Бриллюэна заселены и простые аналитические модели для вычисления теплопроводности не работают.

\section{2. Квантование теплопроводности}

По аналогии с квантованием электронной проводимости

$$
G^{\text {ball }}=\frac{2 q^{2}}{h} M\left(E_{F}\right)
$$

еще 30 лет назад Пендри [25] показал, что должны существовать квантовые ограничения на перенос тепла. Действительно, в уравнении (17) при $T \rightarrow 0$ окно фононной проводимости $W_{p h}(\hbar \omega)$ стремится к $\delta$-функции, так что в пределе

$$
K_{L}=\frac{\pi^{2} k^{2} T}{3 h} T_{p h}(0) M_{p h}(0) .
$$

Для массивных проводников $M_{p h}(\hbar \omega) \rightarrow 0$ при $\hbar \omega \rightarrow 0$, однако, для наноразмерных проводников всегда есть конечное число фононных мод. В случае баллистического транспорта $T_{p h}=1$ и в этом случае можно ожидать что

$$
K_{L}=\frac{\pi^{2} k^{2} T}{3 h} M_{p h} .
$$

Именно этот результат был подтвержден экспериментально на 4-хмодовом нанорезисторе при $T<0.8 K \quad[13] ;$ результаты измерений теплопроводности полностью согласуется с предсказаниями для одномерных баллистических резисторов [26-28].

Квант теплопроводности

$$
g_{0} \equiv \pi^{2} k^{2} T / 3 h
$$

есть максимальная энергия, переносимая в пересчете на одну фононную моду. Удивительно, что квант теплопроводности не зависит от статистики частиц, переносящих тепло, он универсален для фермионов, бозонов и любых аньенов/anyons [29-31].

\section{3. Выводы}

В настоящем обзоре подчеркивается, что концепции, используемые для описания электронного транспорта, с успехом могут быть перенесены на фононный транспорт. И в том и в другом случае подход Ландауэра, обобщенный впоследствии Даттой и Лундстромом, позволяет количественно описать транспортные процессы в проводниках любой размерности и при любых режимах транспорта - от диффузионного до баллистического. Решеточная теплопроводность и электронная проводимость описываются очень схожими по форме уравнениями. Есть, однако, два существенных отличия, связанных с физикой электронных и фононных процессов.

Первое отличие касается ширины зон. В случае электронов ширина зон намного превышает $k T$ и при комнатной температуре заселены лишь уровни вблизи дна зоны проводимости. Ширина фононных дисперсий всего лишь порядка $k T$ и при комнатной температуре все акустические моды в пределах всей зоны Бриллюэна практически заселены. В результате дебаевская модель для акустических мод далеко не столь успешно работает по сравнению с широкой применимостью параболической дисперсии для электронов.

Второе существенное различие связано с тем, что в случае электронов заселенность мод проводимости, контролируемая фермиевским окном проводимости, зависит не только от температуры, как это 
имеет место в случае фононов, но и от положения уровня Ферми. Именно по этой причине электронная проводимость различных материалов может изменяться в очень широких пределах, тогда как теплопроводность изменяется в очень узких пределах всего на несколько порядков.

В основу настоящего обзора положены лекции Марка Лундстрома «Near-Equilibrium Transport: Fundamentals and Applications» [2], Суприе Датты «Fundamentals of Nanoelectronics, Part I: Basic Concepts» [1] и Тимоти Фишера «Thermal Energy at the Nanoscale» [18], прочитанных в 2011-2013 годах в рамках инициативы Purdue University/ nanoHUB-U [www.nanohub.org/u], а также наши статьи $[32,33]$.

\section{4. Благодарности}

Благодарю Н. Е. Кругляк за помощь в работе по подготовке рукописи к печати.

\section{Литература}

1. Supriyo Datta, Lessons from Nanoelectronics: A New Perspective on Transport [Electronic resource] / Hackensack, New Jersey: World Scientific Publishing Company, 2012. - Available at: www.nanohub.org/ courses/FoN1

2. Lundstrom, M. Near-Equilibrium Transport: Fundamentals and Applications [Electronic resource] / M. Lundstrom, C. JeongHackensack. - New Jersey: World Scientific Publishing Company. 2013. - Available at: www.nanohub.org/resources/11763

3. Kruglyak, Yu. Landauer-Datta-Lundstrom Generalized Transport Model for Nanoelectronics [Text] / Yu. Kruglyak // Journal of Nanoscience. - 2014. - Vol. 2015. P. 15. doi: $10.1155 / 2014 / 725420$

4. Kruglyak, Yu. A. A Generalized Landauer-DattaLundstrom Electron Transport Model [Text] / Yu. A. Kruglyak // Russian Journal of Physical Chemistry. - 2014. - Vol. 88, Issue 11. - P. 1826-1836. doi: 10.1134/s0036024414110119

5. Займан, Дж. Электроны и фононы. Теория явлений переноса в твердых телах [Текст] / Дж. Займан. М: ИИЛ, 1962. -488 с.

6. Займан, Дж. Принципы теории твердого тела [Текст] / Дж. Займан. - М: Высшая школа, 1974. - 472 с.

7. Киттель, Ч. Введение в физику твердого тела [Текст] / Ч. Киттель. - М: Наука: 1978. - 791 с.

8. Ашкрофт, Н. Физика твердого тела, тома 1 и 2 [Текст] / Н. Ашкрофт, Н. Мермин. - М: Мир: 1979. - 486 с.

9. Mohr, M. Phonon dispersion of graphite by inelastic x-ray scattering [Text] / M. Mohr, J. Maultzsch, E. Dobardžić, S. Reich, I. Milošević, M. Damnjanović, A. Bosak, M. Krisch, C. Thomsen // Physical Review B. - 2007. - Vol. 76, Issue 3. doi: 10.1103/physrevb.76.035439

10. Елецкий, А. В. Графен: методы получения и теплофизические свойства [Текст] / А. В. Елецкий, И. М. Искандарова, А. А. Книжник, Д. Н. Красиков // Успехи физических наук. - 2011. - Т. 181, № 3. C. 227-258. doi: 10.3367/UFNr.0181.201103a.0233

11. Katsnelson, M. I. Graphene: Carbon in Two Dimensions [Text] / M. I. Katsnelson. - New York: Cambridge University Press, 2012. doi: 10.1017/cbo9781139031080

12. Кругляк, Ю. А. Обобщенная модель электронного транспорта Ландауэра-Датты-Лундстрома [Текст] / Ю. А. Кругляк // Nanosystems, Nanomaterials, Nanotechnologies, 11, № 3: 519 - 549 (2013); Erratum: ibid, 12, № 2: 415 (2014).
13. Schwab, K. Measurement of the quantum of thermal conductance [Text] / K. Schwab, E. A. Henriksen, J. M. Worlock, M. L. Roukes // Nature. - 2000. - Vol. 404. - P. 974.

14. Jeong, C. On Landauer vs. Boltzmann and Full Band vs. Effective Mass Evaluation of Thermoelectric Transport Coefficients [Text] / C. Jeong, R. Kim, M. Luisier, S. Datta, M. Lundstrom // Journal of Applied Physics. - 2010. Vol. 107, Issue 2. - P. 023707. doi: 10.1063/1.3291120

15. Lundstrom, M. Fundamentals of Carrier Transport [Text] / M. Lundstrom. - Cambridge UK: Cambridge University Press, 2012.

16. Jeong, C. Full Dispersion vs. Debye Model Evaluation of Lattice Thermal Conductivity with a Landauer Approach [Text] / C. Jeong, S. Datta, M. Lundstrom // Journal of Applied Physics. - 2011. - Vol. 109. - P. 073718/8. doi: $10.1063 / 1.3567111$

17. Кругляк, Ю. А. Уроки наноэлектроники. 3. Электронная проводимость и моды проводимости в концепции «снизу - вверх» [Текст] / Ю. А. Кругляк, Н. Е. Кругляк // Физическое образование в вузах. - 2013. - Т. 19, № 3. C. $99-110$.

18. Timothy S. Fisher, Thermal Energy at the Nanoscale [Electronic resource] / Hackensack, New Jersey: World Scientific Publishing Company, 2013. - Available at: www.nanohub.org/courses/2

19. Кругляк, Ю. А. Уроки наноэлектроники. 2. Модель упругого резистора и новая формулировка закона Ома в концепции «снизу - вверх» [Текст] / Ю. А. Кругляк, Н. Е. Кругляк // Физическое образование в вузах. - 2013. - Т. 19, № 2. - С. 161-173.

20. Callaway, J. Model for lattice thermal conductivity at low temperatures [Text] / J. Callaway // Physical Review. 1959. - Vol. 113, Issue 4. - P. 1046-1051. doi: 10.1103/ physrev.113.1046

21. Holland, M. G. Analysis of lattice thermal conductivity [Text] / M. G. Holland // Physical Review. 1963. - Vol. 132, Issue 6. - P. 2461-2471. doi: 10.1103/ physrev.132.2461

22. Jeong, C. Thermal conductivity of bulk and thinfilm silicon: a Landauer approach [Text] / C. Jeong, S. Datta, M. Lundstrom // Journal of Applied Physics. -2012. Vol. 111. - P. 093708. doi: 10.1063/1.4710993

23. Gang, C. Nanoscale Energy Transport and Conversion: A Parallel Treatment of Electrons, Molecules, Phonons, and Photons [Text] / C. Gang. - New York: Oxford University Press, 2005. $-560 \mathrm{p}$.

24. Glassbrenner, C. J. Thermal Conductivity of Silicon and Germanium from $3^{\circ} \mathrm{K}$ to the Melting Point [Text] / C. J. Glassbrenner, G. A. Slack // Physical Review. - 1964. Vol. 134, Issue 4A. - P. A1058-A1069. doi: 10.1103/ physrev.134.a1058

25. Pendry, J. B. Quantum limits to the flow of information and entropy [Text] / J. B. Pendry // Journal of Physics A: Mathematical and General. - 1983. - Vol. 16, Issue 10. - P. 2161-2171. doi: 10.1088/0305-4470/16/10/012

26. Angelescu, D. E. Heat transport in mesoscopic systems [Text] / D. E. Angelescu, M. C. Cross, M. L. Roukes // Superlattices and Microstructures. - 1998. -Vol. 23, Issue 3-4. - P. 673-689. doi: 10.1006/spmi.1997.0561

27. Rego, L. G. C. Quantized Thermal Conductance of Dielectric Quantum Wires [Text] / L. G. C. Rego, G. Kirczenow // Physical Review Letters. - 1998. - Vol. 81, Issue 1. - P. 232-235. doi: 10.1103/physrevlett.81.232

28. Blencowe, M. P. Quantum energy flow in mesoscopic dielectric structures [Text] / M. P. Blencowe // Physical Review B. - 1999. - Vol. 59, Issue 7. - P. 4992-4998. doi: 10.1103/physrevb.59.4992 
29. Rego, L. G. C. Fractional exclusion statistics and the universal quantum of thermal conductance: A unifying approach [Text] / L. G. C. Rego, G. Kirczenow // Physical Review B. - 1999. - Vol. 59, Issue 20. -P. 13080-13086. doi: 10.1103/physrevb.59.13080

30. Krive, I. V. Transport properties of quasiparticles with fractional exclusion statistics [Text] / I. V. Krive, E. R. Mucciolo // Physical Review B. - 1999. - Vol. 60, Issue 3. - P. 1429-1432. doi: 10.1103/physrevb.60.1429

31. Caves, C. M. Quantum limits on bosonic communication rates [Text] / C. M. Caves, P .D. Drummond // Reviews of Modern Physics. - 1994. - Vol. 66, Issue 2. P. 481-537. doi: 10.1103/revmodphys.66.481

32. Кругляк, Ю. О. Уроки наноелектроніки. Термоелектричні явища в концепції «знизу - вгору» [Text] / Ю. О. Кругляк, Н. Ю. Кругляк, М. В. Стріха // Sensor Electronics Microsys. Tech. - 2013. - T. 13, № 1. - C. 6-21.

33. Кругляк, Ю. А. Уроки наноэлектроники. 5. Перенос тепла фононами в концепции «снизу - вверх» [Текст] / Ю. А. Кругляк // Физическое образование в вузах. - 2014. - T. 20, № 1. - C. 39-43.

\section{References}

1. Supriyo Datta, Lessons from Nanoelectronics: A New Perspective on Transport (2012). Hackensack, New Jersey: World Scientific Publishing Company. Available at: www.nanohub.org/courses/FoN1

2. Lundstrom, M., Jeong, C. (2013). Near-Equilibrium Transport: Fundamentals and Applications. Hackensack, New Jersey: World Scientific Publishing Company. Available at: www.nanohub.org/resources/11763

3. Kruglyak, Yu. (2014). Landauer-Datta-Lundstrom Generalized Transport Model for Nanoelectronics. Journal of Nanoscience, 2014, 15. doi: 10.1155/2014/725420

4. Kruglyak, Yu. A. (2014). A Generalized LandauerDatta-Lundstrom Electron Transport Model. Russian Journal of Physical Chemistry, 88 (11), 1826-1836. doi: 10.1134/ s0036024414110119

5. Ziman, J. M. (1960). Electrons and phonons. The theory of transport phenomena in solids. Oxford at the Clarendon Press, Oxford, 488.

6. Ziman, J. M. (1964). Principles of the theory of solids. Cambridge University Press, Cambridge, 472.

7. Kittel, C. (1971). Introduction to solid state physics, $4^{\text {th }}$ ed. John Wiley and Sons, New York, 791.

8. Ashcroft, N. W., Mermin, N. D. (1979). Solid State Physics (Philadelphia: Suanders College, 486.

9. Mohr, M., Maultzsch, J., Dobardžić, E., Reich, S., Milošević, I., Damnjanović, M., Bosak, A., Krisch, M., Thomsen, C. (2007). Phonon dispersion of graphite by inelastic x-ray scattering. Physical Review B, 76 (3), 035439/7. doi: 10.1103/physrevb.76.035439

10. Eletskii, A. V., Iskandarova, I. M., Knizhnik, A. A., Krasikov, D. N. (2011). Graphene: fabrication methods and thermophysical properties. Physics Uspekhi, 54, 227-258. doi: 10.3367/UFNe.0181.201103a.0233

11. Katsnelson, M. I. (2012). Graphene: Carbon in Two Dimensions. New York: Cambridge University Press. doi: 10.1017/cbo9781139031080

12. Kruglyak, Yu. A. (2013). The Generalized Landauer-Datta-Lunstrom Electron Transport Model. Nanosystems, Nanomaterials, Nanotechnologies, 11 (3), 519-549. Erratum: ibid, 12 (2), 415.

13. Schwab, K., Henriksen, E. A., Worlock, J. M., Roukes, M. L. (2000). Measurement of the quantum of thermal conductance. Nature, 404, 974.

14. Jeong, C., Kim, R., Luisier, M., Datta, S., Lundstrom, M. (2010). On Landauer vs. Boltzmann and Full
Band vs. Effective Mass Evaluation of Thermoelectric Transport Coefficients. Journal of Applied Physics, 107 (2), 023707. doi: $10.1063 / 1.3291120$

15. Lundstrom, M. (2012). Fundamentals of Carrier Transport. Cambridge UK: Cambridge University Press.

16. Jeong, C., Datta, S., Lundstrom, M. (2011). Full Dispersion vs. Debye Model Evaluation of Lattice Thermal Conductivity with a Landauer Approach. Journal of Applied Physics, 109, 073718/8. doi: 10.1063/1.3567111

17. Kruglyak, Yu. A., Kruglyak, N. E. (2013). Lessons of nanoelectronics. 3. Electronic conductivity and conductivity modes by «bottom - up» approach. Physics in Higher Education, 19 (3), 99-110.

18. Fisher, T. S. (2013). Thermal Energy at the Nanoscale (Hackensack, New Jersey: World Scientific Publishing Company. Available at: www.nanohub.org/ courses/2

19. Kruglyak, Yu. A., Kruglyak, N. E. (2013). Lessons of nanoelectronics. 2. Elastic resistor model and new Ohm's law by «bottom - up» approach. Physics in Higher Education, 19 (2), 161-173.

20. Callaway, J. (1959). Model for lattice thermal conductivity at low temperatures. Physical Review, 113 (4), 1046-1015 doi: 10.1103/physrev.113.1046

21. Holland, M. G. (1963). Analysis of lattice thermal conductivity. Physical Review, 132 (6), 2461-2471. doi: 10.1103/physrev.132.2461

22. Jeong, C., Datta, S., Lundstrom, M. (2012). Thermal conductivity of bulk and thin-film silicon: a Landauer approach. Journal of Applied Physics, 111, 093708. doi: $10.1063 / 1.4710993$

23. Gang, C. (2005). Nanoscale Energy Transport and Conversion: A Parallel Treatment of Electrons, Molecules, Phonons, and Photons. New York: Oxford University Press, 560.

24. Glassbrenner, C. J., Slack, G. A. (1964). Thermal Conductivity of Silicon and Germanium from $3^{\circ} \mathrm{K}$ to the Melting Point. Physical Review, 134 (4A), A1058-A1069. doi: 10.1103/physrev.134.a1058

25. Pendry, J. B. (1983). Quantum limits to the flow of information and entropy. Journal of Physics A: Mathematical and General, 16 (10), 2161. doi: 10.1088/0305-4470/16/10/012

26. Angelescu, D. E., Cross, M. C., Roukes, M. L. (1998). Heat transport in mesoscopic systems, Superlattices and Microstructures, 23 (3-4), 673-689.

doi: 10.1006/spmi.1997.0561

27. Rego, L. G. C., Kirczenow, G. (1998). Quantized Thermal Conductance of Dielectric Quantum Wires. Physical Review Letters, 81 (1), 232-235. doi: 10.1103/ physrevlett.81.232

28. Blencowe, M. P. (1999). Quantum energy flow in mesoscopic dielectric structures. Physical Review B, 59 (7), 4992-4998. doi: 10.1103/physrevb.59.4992

29. Rego, L. G. C., Kirczenow, G. (1999). Fractional exclusion statistics and the universal quantum of thermal conductance: A unifying approach. Physical Review B, 59 (20), 13080-13086. doi: 10.1103/physrevb.59.13080

30. Krive, I. V., Mucciolo, E. R. (1999). Transport properties of quasiparticles with fractional exclusion statistics. Physical Review B, 60 (3), 1429-1432.

doi: 10.1103/physrevb.60.1429

31. Caves, C. M., Drummond, P. D. (1994). Quantum limits on bosonic communication rates, Reviews of Modern Physics, 66 (2), 481-537. doi: 10.1103/revmodphys.66.481

32. Kruglyak, Yu. A., Kruglyak, N. Yu., Strikha, M. V. (2013). Lessons of nanoelectronics. Thermoelectric phenomena in «bottom - up» approach, Sensor Electronics Microsys. Tech., 13 (1), 6-21. 
33. Kruglyak, Yu. A. (2014). Lessons of ach. Physics in Higher Education, 20 (1), 39-43. nanoelectronics. 5. Phonon transport in «bottom - up» appro-

Рекомендовано до публікаиії д-р фіз.-мат. наук Глушков О.В. Дата надходження рукопису 14.01.2015

Кругляк Юрий Алексеевич, доктор химических наук, профессор, кафедра информационных технологий, Одесский государственный экологический университет, ул. Львовская, 15, г. Одесса, Украина, 65016

E-mail: quantumnet@yandex.ua

УДК 537.32

DOI: $10.15587 / 2313-8416.2015 .36443$

\title{
ГРАФЕН В ТРАНСПОРТНОЙ МОДЕЛИ ЛАНДАУЭРА-ДАТТЫ-ЛУНДСТРОМА
}

\author{
(C) Ю. А. Кругляк
}

Обсуждаются такие свойства графена как плотность электронных состояний и носителей тока, число мод и максимальная проводимость, рассеяние и подвижность в графене, циклотронная частота и эффективная масса, плотность фононных состояний, сравнительный вклад электронов и фононов в теплопроводность графена. В справочных целях дается сводка термоэлектрических коэффициентов для графена в баллистическом и диффузионном режимах проводимости со степенным законом рассеяния

Ключевые слова: нанофизика, наноэлектроника, графен, число мод, максимальная проводимость, эффективная масса, фононные состояния, теплопроводность, термоэлектрические коэффициенть

There are discussed the following properties of graphene such as the density of electronic states and current carriers, the number of modes and maximum conductivity, scattering and mobility in graphene, the cyclotron frequency and the effective mass, phonon density of states, the relative contribution of electrons and phonons in the thermal conductivity of graphene. For reference purposes a summary of thermoelectric coefficients for graphene in ballistic and diffusive conduction regimes with the power law of scattering is given

Keywords: nanophysics, nanoelectronics, graphene, mode numbers, maximum conductivity, effective mass, phonon states, thermal conductivity, thermoelectric coefficients

\section{1. Введение}

Модель электронного транспорта ЛандауэраДатты-Лундстрома (ЛДЛ) в режиме линейного отклика сводится к паре транспортных уравнений [1]

$$
\begin{aligned}
& J_{x}=\sigma \frac{d\left(E_{F} / q\right)}{d x}-S \sigma \frac{d T}{d x}\left[A / \mathrm{M}^{2}\right], \\
& J_{Q x}=\pi \sigma \frac{d\left(E_{F} / q\right)}{d x}-\kappa_{0} \frac{d T}{d x}\left[\mathrm{Bm} / \mathrm{M}^{2}\right.
\end{aligned}
$$

или в обращенной форме

$$
\begin{gathered}
\frac{d\left(E_{F} / q\right)}{d x}=\rho J_{x}+S \frac{d T}{d x}, \\
J_{Q x}=\pi J_{x}-\kappa \frac{d T}{d x}
\end{gathered}
$$

c транспортными коэффициентами для 2D проводников

$$
\begin{gathered}
\sigma=1 / \rho=\int \sigma^{\prime}(E) d E \\
\sigma^{\prime}(E)=\frac{2 q^{2}}{h} \frac{M(E)}{W} \lambda(E)\left(-\frac{\partial f_{0}}{\partial E}\right),
\end{gathered}
$$

$$
\begin{aligned}
S & =-\frac{k}{q} \frac{1}{\sigma} \int\left(\frac{E-E_{F}}{k T}\right) \sigma^{\prime}(E) d E=\frac{s_{T}}{\sigma}=\frac{\pi}{T}, \\
\kappa_{0} & =T\left(\frac{k}{q}\right)^{2} \int\left(\frac{E-E_{F}}{k T}\right)^{2} \sigma^{\prime}(E) d E=\kappa+\pi S \sigma .
\end{aligned}
$$

Уравнения (1)-(8) справедливы для диффузионных резисторов любой размерности и любого масштаба, любого типа проводимости, включая биполярную проводимость. Уравнение (6) выписано для 2D проводника; это видно по множителю $M(E) / W$, где $W$ - ширина 2D проводника. При рассмотрении 3D проводников этот множитель нужно заменить на $M(E) / A$, где $A-$ площадь поперечного сечения 3D проводника, а для 1D проводника заменить просто на число мод проводимости $M(E)$. При рассмотрении транспорта во всех режимах от диффузионного до баллистического среднюю длину свободного пробега $\lambda(E)$ нужно заменить на кажущуюся длину по уравнению $1 / \lambda^{a p p}=1 / \lambda+1 / L$, где $L \quad-$ длина проводника. Фактически в этих уравнениях нужно задаться только двумя параметрами, $M(E)$ и $\lambda(E)$. Число мод для проводников любой размерности с параболической дисперсией определяется форму- 